\title{
Measurement of Optical Excitations in Low-Dimensional Materials by Using a Monochromated Electron Source
}

\author{
Ryosuke Senga $^{1}$, Thomas Pichler ${ }^{2}$ and Kazu Suenaga ${ }^{1}$ \\ 1. Nano-Materials Research Institute, National Institute of Advanced Industrial Science and Technology \\ (AIST), Tsukuba, Japan \\ 2. Faculty of Physics, University of Vienna, Vienna, Austria
}

Optical properties of low-dimensional materials are strongly governed by excitons and their interactions with defects. For instance, non-radiative exciton decays in carbon nanotubes are presumably happened at defects and result in the low luminescence quantum yield [1]. On the other hand, specific defects in low-dimensional materials including carbon nanotubes, hexagonal boron nitrides and transition metal dichalcogenides modify their electronic structures and serve as dominant emission sites [2,3]. Thus the direct correlation between locally modulated exciton behaviors and atomic structures is necessary for further understandings. However the conventional light-probe emission/absorption spectroscopy has the inferior spatial resolution to assign an individual defect. For such local optical measurements, an electron probe in a transmission electron microscope (TEM), which can picture the atomic structures of materials and also induce optical excitations in single quantum objects, is quite useful. In addition, the recent development of a monochromator for TEM has pushed up the electron energy-loss spectroscopy (EELS) energy resolution to better than a few tens of millielectron volts, allowing us to identify the absorption peaks for optical excitation involving the quantum effects [4-8].

In this study, the valence-loss spectra including the optical gap transitions of individual semiconducting carbon nanotubes were obtained by using a JEOL TEM system (3C2) equipped with a Schottky field emission gun, a double Wien filter monochromator, and delta correctors at $60 \mathrm{keV}$. EEL spectra were collected by STEM mode, in which the energy resolution was set to $30 \mathrm{meV}$ in FWHM, with a Gatan Quantum spectrometer modified for low primary energy operation.

We first compered the EEL spectra of semiconducting nanotubes to the optical absorption spectra taken from a single chirality suspension prepared by a gel column chromatography [9]. EEL spectra were taken from a single isolated $(9,2)$ SWNT (freestanding in vacuum). Although the EEL spectra and the optical absorption spectra show the similar well-separated excitonic peaks corresponding to the transitions between van Hove singularities, their selection rules are different depending on the polarization condition (Fig. 1(a)). For the conventional optical absorption spectroscopy of carbon nanotube, only $E_{i i}(i=1,2,3 \ldots)$ transitions are allowed. This is comparable to the EEL spectra taken by an aloof geometry where dipolar induced transitions are dominant (not shown). On the other hand, EELS with a near-field condition in which an electron beam directly hits on a SWNT with a large convergence angle (Fig. 1(b)) exhibits the multipolar-induced transitions. Therefore, the EEL spectrum shows $E_{i j}(i \neq j)$ transitions, which are basically forbidden in a typical optical absorption spectrum (Fig. 1(c)). The exciton phonon coupling or direct band-to-band transitions were also observed in the EEL spectra. Such detailed information related to the joint density of states is beneficial for understanding the optical properties of individual carbon nanotubes.

Moreover, the EEL spectrum taken from a certain type of defect presents a characteristic modification near the lowest excitation peak. The line-width of exciton peak shows a variety of broadening at 
different defect sites and suggests different degrees of shortening of its lifetime. Such a direct correlation between excitonic behaviors and atomic structures in a quantum object would be useful for further study to quantify the optoelectronic behaviors of nanoscale devices.

\section{References}

[1] J. J. Crochet et al., Nature Nanotech. 7 (2012), 126.

[2] Y. Miyauchi et al., Natute Photo. 7 (2013), 715.

[3] R. Bourrellier et al., Nano Lett. 16 (2016), 4317.

[4] L. H. G. Tizei et al., Phys. Rev. Lett. 114 (2015), 107601.

[5] J. Lin et al., Nano Lett. 16 (2016), 7198.

[6] Y. Sato and M. Terauchi, Microsc. Microanal. 20 (2014), 807.

[7] R. Senga et al., Nano Lett. 16 (2016), 3661.

[8] F. S. Hage et al., Phys. Rev. B 95 (2017), 195411.

[9] X. Wei et al., Nature Comm. 7 (2016), 12899.

[9] RS acknowledge funding from JSPS KAKENHI, 17H04797. Dr. H Kataura is thanked for their sample preparations.

(a)

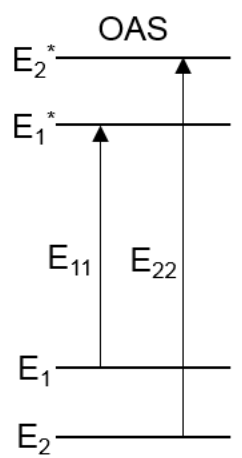

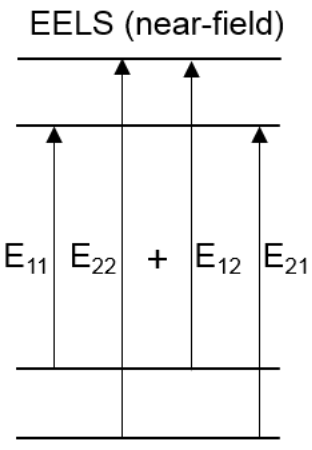

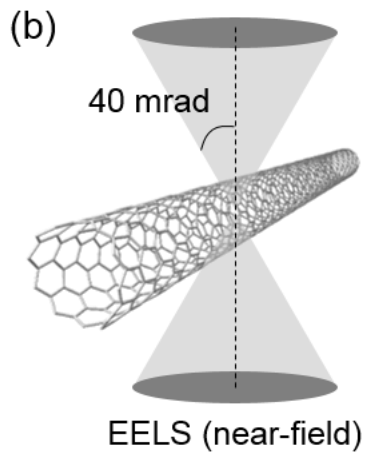

(c)

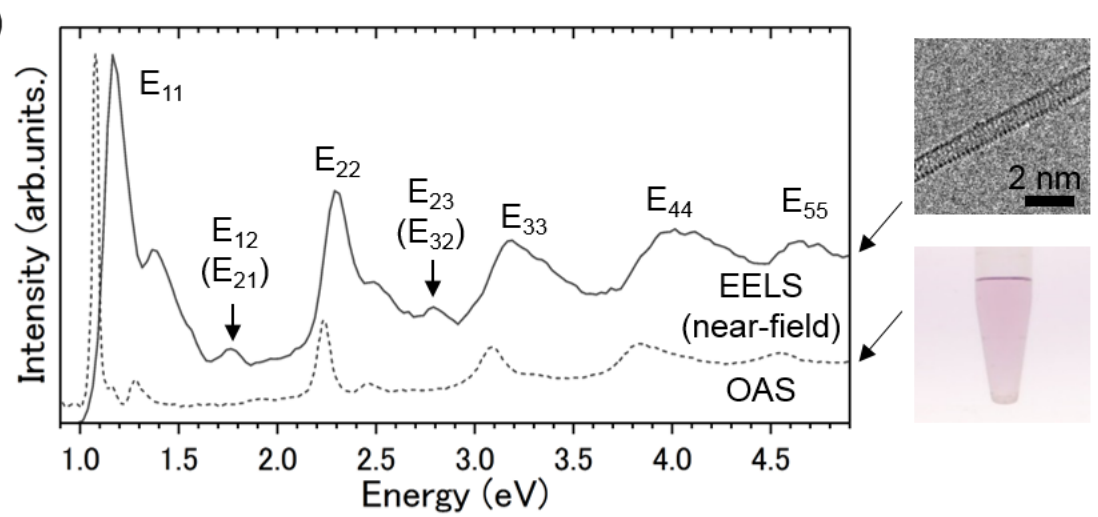

Figure 1. Comparison between electron energy loss spectroscopy (EELS) and optical absorption spectroscopy (OAS) of semiconducting carbon nanotubes. (a) The allowed transitions in OAS and EELS. The selection rule in EELS corresponds to the polarization of incident beam. When the converged electron probe directly hit the nanotube (near-field) as shown in (b), multipolar induced transitions including $E_{i j}(i \neq j)$ transition, which are basically forbidden in OAS, are involved in the EEL spectrum as shown in (c). 